\title{
STOCHASTIC REPRESENTATION \\ AND SINGULARITIES OF SOLUTIONS \\ OF SECOND ORDER EQUATIONS \\ WITH SEMIDEFINITE CHARACTERISTIC FORM
}

BY

KAZUO AMANO

\begin{abstract}
In the theory of partial differential equations, there is no explicit representation of solutions for general degenerate elliptic-parabolic equations. However, Stroock and Varadhan [15] have obtained a stochastic representation for such a wider class of equations in $L^{\infty}$ space. In this paper we establish, by using Stroock and Varadhan's stochastic representation, a method which enables us to construct solutions with singularities of second order equations with semidefinite characteristic form. Our theorems are not probabilistic paraphrases of the results obtained in the theory of partial differential equations. In fact, each assumption of the theorems is much weaker than any assumption of corresponding known results.
\end{abstract}

Introduction. As is well known, it is one of many interesting problems to characterize the (analytic) singularities of solutions of second order equations with semidefinite characteristic form, and this problem was investigated by many authors. However, most of them gave only sufficient conditions for (analytic-) hypoellipticity. In this paper we shall give sufficient conditions under which a certain class of second order operators with semidefinite characteristic form are not (analytic-) hypoelliptic. The proof depends on Stroock-Varadhan's stochastic representation of solutions of degenerate elliptic-parabolic equations; we solve the Dirichlet problem with singular boundary condition by using probabilistic methods and obtain a solution with (analytic) singularities.

Let $G$ be an open set in $\mathbf{R}^{d}$ and let us consider a differential operator

$$
A=\sum_{i, j=1}^{d} a_{i j}(x) \frac{\partial^{2}}{\partial x_{i} \partial x_{j}}+\sum_{i=1}^{d} b_{i}(x) \frac{\partial}{\partial x_{i}}+c(x)
$$

with real coefficients belonging to $C^{\infty}(G)$. Without loss of generality we may assume $a_{i j}=a_{j i}$. Let $X_{0}, X_{1}, \ldots, X_{d}$ be vector fields defined by

$$
\begin{gathered}
X_{0}=\sum_{i=1}^{d}\left(b_{i}-\sum_{j=1}^{d} \frac{\partial a_{i j}}{\partial x_{j}}\right) \frac{\partial}{\partial x_{i}}, \\
X_{i}=\sum_{j=1}^{d} a_{i j} \frac{\partial}{\partial x_{j}} \quad(i=1,2, \ldots, d) .
\end{gathered}
$$

Received by the editors October 25, 1983.

1980 Mathematics Subject Classification. Primary 35J15; Secondary 35J25.

Key words and phrases. Degenerate elliptic-parabolic equations.

(C) 1984 American Mathematical Society $0002-9947 / 84 \$ 1.00+\$ .25$ per page 
THEOREM 1. Assume that

$$
\sum_{i, j=1}^{d} a_{i j}(x) \xi_{i} \xi_{j} \geq 0 \text { for all }(x, \xi) \in G \times \mathbf{R}^{d}
$$

and assume that there is a submanifold $M$ of $G$ such that

$$
\sum_{i, j=1}^{d}\left|a_{i j}\right|+\sum_{i=1}^{d}\left|b_{i}\right| \neq 0 \text { on } M
$$

and

$$
X_{i}(x) \in T_{x} M \quad \text { for all } x \in M \text { and } i=0,1, \ldots, d .
$$

Then for any point $p$ on $M$ there exist an open neighborhood $U$ of $p$ in $G$ and $a$ function $u$ of the class $L_{\mathrm{loc}}^{1}(U)$ such that $A u=0$ in $U$ and

$$
\left(p, \xi^{0}\right) \in W F(u) \text { for some } \xi^{0} \in\left(T_{p}^{*} M\right)^{\perp} \backslash 0,
$$

where $\left(T_{p M}^{*}\right)^{\perp}=\left\{\xi \in T_{p}^{*} G:\langle\xi, \eta\rangle=0\right.$ for any $\left.\eta \in T_{p} M\right\}$.

We cannot remove condition (2). In fact, the operators

$$
|x|^{2 k} \sum_{i=1}^{d}\left(\frac{\partial}{\partial x_{i}}\right)^{2}-1 \quad(k=2,3, \ldots), \quad \exp \left(\frac{-1}{|x|}\right) \sum_{i=1}^{d}\left(\frac{\partial}{\partial x_{i}}\right)^{2}-1
$$

are hypoelliptic in $\mathbf{R}^{d}$ (cf. $[\mathbf{8}, \mathbf{2}]$ ). For any point $p$ in $G$ there passes an integral manifold $M$ through the point $p$ which satisfies condition (3). In fact, let $\Delta$ be a distribution (in the sense of differential geometry) spanned by the vector fields $X_{0}, X_{1}, \ldots, X_{d}$, i.e., $\Delta$ is a mapping defined in $G$ such that

$$
\Delta(x)=\left\{\sum_{i=0}^{d} \lambda_{i} X_{i}(x): \lambda_{i} \in \mathbf{R}(i=0,1, \ldots, d)\right\} .
$$

$\mathfrak{G}$ denotes the group of local $C^{\infty}$-diffeomorphisms in $G$ generated by $X_{0}, X_{1}, \ldots, X_{d}$. Let $D$ be the smallest $\mathfrak{G}$-invariant distribution in $G$, i.e., $D(x)$ is the linear hull of all vectors $v \in \mathbf{R}^{d}$ such that $v \in \Delta(x)$ or $v \in d \phi(\Delta(y))$ for some $y \in G$ and some $\phi \in \mathfrak{G}$ satisfying $x=\phi(y)$. Then, according to Sussmann [16], through every point in $G$ there passes a maximal integral manifold of $D$. Any maximal integral manifold of $D$ satisfies condition (3). Consequently, we obtain the following fact: If the operator $A$ is hypoelliptic in $G$, then

$$
\operatorname{dim} D(x)=d \quad \text { in } G .
$$

It is to be noted that $L(x) \subset D(x)$ in $G$ and further, if $X_{0}, X_{1}, \ldots, X_{d}$ are analytic vector fields, then $L(x)=D(x)$ in $G$, where

$$
L(x)=\left\{X(x): X \in \operatorname{Lie}\left(X_{0}, X_{1}, \ldots, X_{d}\right)\right\} .
$$

However, (5) is not a sufficient condition for hypoellipticity. In fact, Kusuoka and Stroock $[\mathbf{1 1}]$ proved that the operator

$$
\left(\frac{\partial}{\partial x}\right)^{2}+\exp \left(\frac{-1}{|x|^{k}}\right)\left(\frac{\partial}{\partial y}\right)^{2}+\left(\frac{\partial}{\partial z}\right)^{2} \quad(k>1)
$$

is not hypoelliptic in $\mathbf{R}^{3}$. 
In case the coefficients $a_{i j}, b_{i}, c$ are real analytic, Derridj [5] and Oleinnik and Radkevic [12] have proved that on the same assumption of Theorem 1 there exists a solution $u$ of the equation $A u=0$ with sing supp $u \neq \emptyset$. Amano $[1]$ has proved that their theorem remains true when the coefficients are real $C^{\infty}$-smooth.

THEOREM 2. Assume that

$$
\sum_{i, j=1}^{d} a_{i j}(x) \xi_{i} \xi_{j} \geq 0 \quad \text { for all }(x, \xi) \in G \times \mathbf{R}^{d}
$$

and assume that there is a real valued function $\psi$ belonging to the class $C^{\infty}(G)$ such that $\psi$ attains 0 at some point in $G, \nabla \psi \neq 0$ in $G$,

$$
\sum_{i, j=1}^{d} a_{i j}(x) \frac{\partial \psi}{\partial x_{i}}(x) \frac{\partial \psi}{\partial x_{j}}(x)\left(\begin{array}{cc}
=0 & \text { if } \psi(x)=0 \\
>0 & \text { if } \psi(x) \neq 0
\end{array}\right.
$$

and

$$
\left\langle X_{0}, \nabla \psi\right\rangle \neq 0 \text { on } M,
$$

where $M=\{x \in G: \psi(x)=0\}$. Then for any point $p$ on $M$ there exist an open neighborhood $U$ of $p$ in $G$ and a function $u$ of the class $L^{\infty}(U)$ such that $A u=0$ in $U$ and

$$
\left(p, \xi^{0}\right) \in W F_{A}(u) \quad \text { for some } \xi^{0} \in\left(T_{p}^{*} M\right)^{\perp} \backslash 0 .
$$

Condition (7) means that $M$ is a characteristic hypersurface of the operator $A$. Condition (8) is not removable. In fact, we have only to consider an operator $A=\partial / \partial x_{d}$ in $\mathbf{R}^{d}$ and a hyperplane $M=\left\{x \in \mathbf{R}^{d}: x_{d}=0\right\}$. When (9) is not satisfied, the result of Theorem 2 is not always true. However, if we assume

$$
\left\langle X_{0}, \nabla \psi\right\rangle=0 \text { and } X_{0} \neq 0 \text { on } M
$$

instead of (9), then, for any point $p$ on $M$, Theorem 1 ensures the existence of a solution $u$ of the equation $A u=0$ satisfying (4). It is to be noted that we can apply Theorem 2 to degenerate parabolic operators.

THEOREM 3. Assume $d \geq 2$ and assume that there is a real valued function $\psi$ belonging to the class $C^{\infty}(G)$ such that $\psi$ attains 0 at some point in $G, \nabla \psi \neq 0$ in $G$,

$$
\sum_{i, j=1}^{d} a_{i j}(x) \xi_{i} \xi_{j}\left(\begin{array}{ll}
\geq 0 & \text { if } \psi(x) \geq 0 \text { and } \xi \in \mathbf{R}^{d} \\
\leq 0 & \text { if } \psi(x) \leq 0 \text { and } \xi \in \mathbf{R}^{d}
\end{array}\right.
$$

and

$$
X_{0}(x) \neq 0 \text { on } M,
$$

where $M=\{x \in G: \psi(x)=0\}$.

(i) If $\left\langle X_{0}, \nabla \psi\right\rangle \equiv 0$ on $M$ and if

$$
\sum_{i, j=1}^{d} a_{i j}(x) \frac{\partial \psi}{\partial x_{i}}(x) \frac{\partial \psi}{\partial x_{j}}(x) / \psi(x) \rightarrow 0 \quad \text { as } \psi(x) \rightarrow 0
$$


then for any point $p$ on $M$ there exist an open neighborhood $U$ of $p$ in $G$ and a function $u$ of the class $L^{\infty}(U)$ such that $A u=0$ in $U$ and

$$
\left(p, \xi^{0}\right) \in W F(u) \quad \text { for some } \xi^{0} \in\left(T_{p}^{*} M\right)^{\perp} \backslash 0 .
$$

(ii) If $\left\langle X_{0}, \nabla \psi\right\rangle<0$ on $M$, then for any point $p$ on $M$ there exist an open neighborhood $U$ of $p$ in $G$ and a function $u$ of the class $L^{\infty}(U)$ such that $A u=0$ in $U$ and

$$
p \in \operatorname{sing} \operatorname{supp} u \text {. }
$$

Zuily [17] has proved that if $a_{i j}(x)$ are real analytic and if the matrix $\left(a_{i j}(x)\right)_{d \times d}$ is either positive or negative semidefinite at each point $x$ in $G$, then for any point $p$ in $G$ there exist an open neighborhood $V$ of $p$ in $G$ and a real analytic function $\psi(x)$ in $V$ such that (11) is valid in $V$. If $\left\langle X_{0}, \nabla \psi\right\rangle>0$ on $M$, then the operator $A$ is hypoelliptic on a moderate assumption ( $\mathrm{cf} .[\mathbf{1 7}, \mathbf{3}])$. It is to be noted that Theorem 3 shows that the operators

$$
x_{d}^{k} \sum_{i=1}^{d}\left(\frac{\partial}{\partial x_{i}}\right)^{2}-\frac{\partial}{\partial x_{d}} \quad(k \text { is odd, } d \geq 2)
$$

are not hypoelliptic in $\mathbf{R}^{d}$ and on the other hand, Zuily's theorem [17] shows that the operators

$$
x_{d}^{k} \sum_{i=1}^{d}\left(\frac{\partial}{\partial x_{i}}\right)^{2}+\frac{\partial}{\partial x_{d}} \quad(k \text { is odd, } d \geq 2)
$$

are hypoelliptic in $\mathbf{R}^{d}$. Thus the sign of $\left\langle X_{0}, \nabla \psi\right\rangle$ has a significant influence upon the hypoellipticity in case the characteristic form changes sign.

When $a_{i j}(x) / \psi(x) \in C^{\infty}(G), \operatorname{rank}\left(a_{i j}(x) / \psi(x)\right)_{d \times d}>0$ in $G$ and

$$
\sum_{i, j=1}^{d} a_{i j}(x) \frac{\partial \psi}{\partial x_{i}}(x) \frac{\partial \psi}{\partial x_{j}}(x) / \psi(x) \equiv 0 \quad \text { in } G
$$

Beals and Fefferman $[3]$ have proved that on the same assumption of Theorem 3 there exists a solution $u$ of the equation $A u=f$ such that sing supp $f \varsubsetneqq \operatorname{sing}$ supp $u$. Helffer and Zuily [7] have proved that the operators of Fuchs type are not hypoelliptic. Kannai $[\mathbf{1 0}]$ has given a virtually complete characterization of hypoelliptic ordinary differential operators.

THEOREM 4. Assume that there is a real-valued function $\psi$ belonging to the class $C^{\infty}(G)$ such that $\psi$ attains 0 at some point in $G, \nabla \psi \neq 0$ in $G$ and

$$
\sum_{i, j=1}^{d} a_{i j}(x) \xi_{i} \xi_{j}\left(\begin{array}{ll}
\geq 0 & \text { if } \psi(x) \geq 0 \text { and } \xi \in \mathbf{R}^{d} \\
\leq 0 & \text { if } \psi(x) \leq 0 \text { and } \xi \in \mathbf{R}^{d}
\end{array}\right.
$$

and assume that

$$
\sum_{i, j=1}^{d} a_{i j}(x) \frac{\partial \psi}{\partial x_{i}}(x) \frac{\partial \psi}{\partial x_{j}}(x) \neq 0 \quad \text { in } G \backslash M
$$


where $M=\{x \in G: \psi(x)=0\}$. If $\left\langle X_{0}, \nabla \psi\right\rangle<0$ on $M$, then for any point $p$ on $M$ there exist an open neighborhood $U$ of $p$ in $G$ and a function $u$ of the class $L^{\infty}(U)$ such that $A u=0$ in $U$ and

$$
\left(p, \xi^{0}\right) \in W F_{A}(u) \quad \text { for some } \xi^{0} \in\left(T_{p}^{*} M\right)^{\perp} \backslash 0 .
$$

1. Preliminaries. In this section we assume that the coefficients $a_{i j}, b_{i}, c$ are real valued functions such that $a_{i j} \in C_{\mathrm{bdd}}^{2}\left(\mathbf{R}^{d}\right), b_{i} \in C_{\mathrm{bdd}}^{\mathbf{1}}\left(\mathbf{R}^{d}\right)$ and $c \in C_{\mathrm{bdd}}\left(\mathbf{R}^{d}\right)$, i.e., $\partial^{\alpha} a_{i j}, \partial^{\beta} b_{i}$ and $c$ are bounded continuous functions in $\mathbf{R}^{d}$ for $|\alpha| \leq 2,|\beta| \leq 1$, and assume that

$$
\sum_{i, j=1}^{d} a_{i j}(x) \xi_{i} \xi_{j} \geq 0 \text { for all }(x, \xi) \in \mathbf{R}^{d} \times \mathbf{R}^{d}
$$

For brevity we set

$$
a(x)=\left(a_{i j}(x): \begin{array}{c}
i \downarrow 1 \ldots d \\
j \rightarrow 1 \ldots d
\end{array}\right), \quad b(x)=\left(b_{1}(x), \ldots, b_{d}(x)\right)
$$

and

$$
\sigma(x)=\left(\sigma_{i j}(x): \begin{array}{l}
i \downarrow 1 \ldots d \\
j \rightarrow 1 \ldots d
\end{array}\right)=(2 a(x))^{1 / 2}
$$

LEMMA $1.1[\mathbf{1 3}] . \sigma_{i j}(x)$ are Lipschitz continuous in $\mathbf{R}^{d}$.

Let $\Omega$ be the space of all $\mathbf{R}^{d}$-valued continuous functions defined on $[0, \infty)$. $x(t)=x(t, \omega)=\omega(t)$ denotes the value of a function $\omega \in \Omega$ at $t$. $\mathfrak{F}_{t}$ is the $\sigma$-field generated by the functions $x(s, \cdot)$ for $0 \leq s \leq t$. $\mathfrak{F}$ is the smallest $\sigma$-field containing $\mathfrak{F}_{t}$ for all $t \geq 0$.

The following lemma is well known.

LEMMA 1.2. There exists a probability measure $P$ defined on $(\Omega, \mathfrak{F})$ such that $\left(\Omega, \mathfrak{F}, \mathfrak{F}_{t}, x(t), P\right), t \geq 0$, is a Brownian motion.

For convenience we often rewrite the function $x(t)=x(t, \omega)$ as $w(t)=w(t, \omega)$ when $\left(\Omega, \mathfrak{F}, \mathfrak{F}_{t}, x(t), P\right), t \geq 0$, is a Brownian motion.

The following two lemmas are also well known.

LEMMA 1.3. Let $\left(\Omega, \mathfrak{F}, \mathfrak{F}_{t}, w(t), P\right), t \geq 0$, be a Brownian motion. Then for any $x \in \mathbf{R}^{d}$ and any $T>0$ there exists a unique solution, say $\xi_{x}(t)$, of the initial value problem

$$
\begin{aligned}
& d \xi=\sigma(\xi) d w(t)+b(\xi) d t \\
& \xi(0)=x \quad P \text {-a.s. }
\end{aligned}
$$

in $M_{w}^{2}[0, T]$. Here $M_{w}^{2}[0, T]$ is the set of all nonanticipative functions $f(t)$ with respect to $\left(\Omega, \mathfrak{F}_{t}\right)$ satisfying $E\left[\int_{0}^{T}|f(t)|^{2} d t\right]<+\infty$.

LEMMA 1.4. By

$$
P_{x}(F)=P\left[\left\{\omega \in \Omega: \xi_{x}(\cdot, \omega) \in F\right\}\right] \quad(F \in \mathfrak{F})
$$

we define a probability measure $P_{x}$ on $(\Omega, \mathfrak{F})$. Then $\left(\Omega, \mathfrak{F}, \mathfrak{F}_{t}, x(t), P_{x}\right), t \geq 0, x \in$ $\mathbf{R}^{d}$, is a diffusion process generated by the differential operator $A-c(x)$.

The proofs of our theorems essentially depend on Itô's formula (Lemma 1.5) and Stroock and Varadhan's stochastic representation (Lemma 1.6). 
LEMMA 1.5 (ITÔ'S FORMULA). If $\xi(t)$ is a solution of the stochastic differential equation (1.1), then

$$
\begin{aligned}
d\left[v(\xi(t)) \exp \left\{\int_{0}^{t} c(\xi(s)) d s\right\}\right]= & \nabla v(\xi(t)) \exp \left\{\int_{0}^{t} c(\xi(s)) d s\right\} \sigma(\xi(t)) d w(t) \\
& +A v(\xi(t)) \exp \left\{\int_{0}^{t} c(\xi(s)) d s\right\} d t
\end{aligned}
$$

$P$-a.s. for any $v \in C^{2}\left(\mathbf{R}^{d}\right)$.

Let $U$ be an open set in $\mathbf{R}^{d}$ with $C^{2}$ boundary $\partial U . \tau \leftleftarrows$ denotes the exit time of $x(t)$ from $\bar{U}$. $\Gamma$ and $\Sigma$ are the subsets of $\partial U$ such that

$$
\Gamma=\left\{x \in \partial U: P_{x}(\tau>0)=0\right\}
$$

and

$$
\Sigma=\left\{x \in \partial U:\langle a(x) \nu(x), \nu(x)\rangle>0 \text { or }\left\langle X_{0}(x), \nu(x)\right\rangle<0\right\},
$$

where $\nu(x)$ is the inward normal vector at $x$ to $\partial U$. The sets $\Gamma$ and $\Sigma$ are not essentially different; in fact $\Sigma \subset \Gamma \subset \bar{\Sigma}$ (cf. [15]). $C$ is a constant defined by

$$
C=\sup _{x \in U} c(x) \vee 0 .
$$

Then we have the following

LEMMA 1.6 [15]. Assume that

$$
\sup _{x \in U} E_{x}\left[(1+\tau) e^{C \tau}\right]<+\infty .
$$

Then for given $f \in L^{\infty}(U)$ and $g \in L^{\infty}(\Gamma) \cap C(\Sigma)$, the function

$$
u(x)=E_{x}\left[g(x(\tau)) \exp \left\{\int_{0}^{\tau} c(x(s)) d s\right\}-\int_{0}^{\tau} f(x(t)) \exp \left\{\int_{0}^{t} c(x(s)) d s\right\} d t\right]
$$

is a unique solution of the Dirichlet problem

$$
\begin{aligned}
& A u=f \quad \text { in } U, \\
& \lim _{\substack{x \rightarrow a \\
x \in U}} u(x)=g(a) \quad(a \in \Sigma)
\end{aligned}
$$

in $L^{\infty}(U)$. Here (1.4) means

$$
\int u A^{*} \phi d x=\int f \phi d x \quad\left(\phi \in C_{0}^{\infty}\left(\mathbf{R}^{d}\right)\right) .
$$

In case $f=0$, we can replace the assumption (1.3) by

$$
\sup _{x \in U} E_{x}\left[e^{C \tau}\right]<+\infty
$$

(1.3) is fulfilled, if the diameter of $U$ is sufficiently small. In fact, we have the following lemma. 
LEMMA $1.7[\mathbf{1}]$. Let $U_{\rho}$ be an open neighborhood of a fixed point $p$ in $\mathbf{R}^{d}$, with diameter $U_{\rho}=\rho$, and let $\tau_{\rho}$ be the exit time of $x(t)$ from $\bar{U}$. If

$$
\sum_{i, j=1}^{d}\left|a_{i j}(p)\right|+\sum_{i=1}^{d}\left|b_{i}(p)\right| \neq 0
$$

then

$$
\varlimsup_{\rho \downarrow 0} \sup _{x \in U} E_{x}\left[e^{C \tau_{\rho}}\right]<+\infty
$$

and

$$
\lim _{\rho \backslash 0} \sup _{x \in U} E_{x}\left[\left(\tau_{\rho} e^{c \tau_{\rho}}\right)^{k}\right]=0
$$

for any constant $C$ and any $k=1,2, \ldots$

In the proofs of our theorems we use fundamental properties of the probability measures $P_{x}$ (Lemmas 1.8 and 1.9).

Let us define $S(x)$ to be the set of $\omega \in \Omega$ such that

$$
\omega(t)=x+\int_{0}^{t} X_{0}(\omega(s)) d s+\sum_{i=1}^{d} \int_{0}^{t} \psi_{i}(s) X_{i}(\omega(s)) d s
$$

for some bounded measurable functions $\psi_{i}:[0, \infty) \rightarrow \mathbf{R}(i=1, \ldots, d) . \bar{S}(x)$ denotes the closure of $S(x)$ with respect to the topology of the space $\Omega$.

LEMMA $1.8[15] . \operatorname{supp}\left(P_{x}\right)=\bar{S}(x)$.

LEMMA 1.9 [6]. Let $V$ be an open set in $\mathbf{R}^{d}$ and let $K$ be a compact set of $\bar{V}$. Assume that there exists a function $w \in C^{2}(V \backslash K)$ and a constant $\gamma \geq 0$ such that

$$
(A-c) w \leq \gamma w \text { in } V \backslash K
$$

and

$$
w(x) \rightarrow+\infty \quad \text { if } x \in V \backslash K, d(x, K) \rightarrow 0 .
$$

Then

$$
P_{x}[x(t) \in K \text { for some } 0 \leq t<\sigma]=0
$$

for any $x \in V \backslash K$, where $\sigma$ is the exit time of $x(t)$ from $V$.

2. Proof of Theorem 1. Throughout this section, we assume (1), and assume that $M$ is a submanifold of $G$ satisfying (2) and (3). Let $p$ be a point on $M$ and let $V$ be an open neighborhood of $p$ in $G$. Let us take a nonnegative function $\chi \in C_{0}^{\infty}(V)$ and consider a diffusion process $\left(\Omega, \mathfrak{F}, \mathfrak{F}_{t}, x(t), P_{x}\right)$ generated by the operator $\chi(A-c(x))$ (cf. Lemma 1.4). Then we have the following two lemmas.

LEMMA 2.1. If the diameter of $V$ is sufficiently small, then

$$
P_{x}[x(t) \in \bar{M} \text { for some } t \geq 0]=0
$$

for all $x \in \mathbf{R}^{d} \backslash \bar{M}$.

ProOF. Without loss of generality, we may assume that

$$
\bar{M} \cap V=\left\{x \in V: x_{r+1}=\cdots=x_{d}=0\right\} \quad(0 \leq r<d) .
$$


By (1) and (3), we have

$$
a_{i j}(x)=O\left(\left|x^{\prime \prime}\right|^{2}\right), \quad b_{i}(x)=O\left(\left|x^{\prime \prime}\right|\right)
$$

for $r+1 \leq i, j \leq d$ when $x \in V \backslash \bar{M}$ and $d(x, \bar{M}) \rightarrow 0$, where $x^{\prime \prime}=\left(x_{r+1}, \ldots, x_{d}\right)$. It is easy to show that $w(x)=1 /\left|x^{\prime \prime}\right|^{\varepsilon}(\varepsilon>0)$ satisfies conditions (1.7) and (1.8) for $K=\bar{M} \cap \bar{V}$. Hence, by Lemma 1.9 ,

$$
P_{x}[x(t) \in \bar{M} \text { for some } 0 \leq t<\sigma]=0
$$

for all $x \in V \backslash \bar{M}$, where $\sigma$ is the exit time of $x(t)$ from $V$. On the other hand, by Lemma 1.8, we have $\sigma=+\infty P_{x}$-a.s. if $x \in V$, and

$$
P_{x}[x(t)=x \text { for all } t \geq 0]=1 \text { if } x \notin V .
$$

Therefore, we obtain (2.1).

LEMMA 2.2. If the diameter of $M$ is sufficiently small, then

$$
\begin{aligned}
d[v(x(t)) \exp & \left.\left\{\int_{0}^{t} \chi c(x(s)) d s\right\}\right] \\
= & \nabla v(x(t)) \chi^{1 / 2} \sigma(x(t)) \exp \left\{\int_{0}^{t} \chi c(x(s)) d s\right\} d w(t) \\
& +\chi A v(x(t)) \exp \left\{\int_{0}^{t} \chi c(x(s)) d s\right\} d t
\end{aligned}
$$

$P_{x}$-a.s. for any $v \in C^{2}\left(\mathbf{R}^{d} \backslash \bar{M}\right)$ and any $x \in \mathbf{R}^{d} \backslash \bar{M}$.

Proof. For a fixed $v \in C^{2}\left(\mathbf{R}^{d} \backslash \bar{M}\right)$ let us take a function $v_{\varepsilon} \in C^{2}\left(\mathbf{R}^{d}\right), \varepsilon>0$, such that $v_{\varepsilon}(x)=v(x)$ when $d(x, \bar{M})>\varepsilon$. For $T>0$ and $\varepsilon>0$ we set

$$
\begin{aligned}
& F_{T, \varepsilon}=\{\omega \in \Omega: d(x(t), \bar{M})>\varepsilon \text { for all } 0 \leq t \leq T\}, \\
& F_{T}=\{\omega \in \Omega:(2.3) \text { is valid for all } 0 \leq t \leq T\}
\end{aligned}
$$

By Lemma 1.5, we have

$$
\begin{aligned}
d\left[v_{\varepsilon}(x(t)) \exp \right. & \left.\left\{\int_{0}^{t} \chi c(x(s)) d s\right\}\right] \\
= & \nabla v_{\varepsilon}(x(t)) \chi^{1 / 2} \sigma(x(t)) \exp \left\{\int_{0}^{t} \chi c(x(s)) d s\right\} d w(t) \\
& +\chi A v_{\varepsilon}(x(t)) \exp \left\{\int_{0}^{t} \chi c(x(s)) d s\right\} d t
\end{aligned}
$$

$P_{x}$-a.s. for any $x \in \mathbf{R}^{d}$. Hence, for any $x \in \mathbf{R}^{d}$ there is a set $N_{\varepsilon}^{x} \in \mathfrak{F}$ such that $P_{x}\left(N_{\varepsilon}^{x}\right)=0$ and $F_{T, \varepsilon} \subset F_{T} \cup N_{\varepsilon}^{x}$. Since, by Lemma 2.1,

$$
P_{x}\left(\bigcup_{n=1}^{\infty} F_{T, 1 / n}\right)=1 \text { for } x \in \mathbf{R}^{d} \backslash \bar{M}, T>0
$$

we have

$$
P_{x}\left(F_{T}\right)=1 \text { for } x \in \mathbf{R}^{d} \backslash \bar{M}, T>0 .
$$

This completes the proof of Lemma 2.2. 
LEMMA $2.3[1]$. Let $\left(\Omega, \mathfrak{F}, \mathfrak{F}_{t}, w(x), P\right)$ be a Brownian motion and let $\tau$ be a stopping time such that $0 \leq \tau<\infty P$-a.s. Then

$$
E\left[\sup _{0 \leq t \leq \tau}\left|\int_{0}^{t} f(s) d w(s)\right|^{2}\right] \leq 4 E\left[\int_{0}^{\tau}|f(s)|^{2} d s\right]
$$

if $f(t) \in M_{w}^{2}[0, T]$ for any $T>0$. that

Proposition 2.4. Assume that $g \in C^{2}(G \backslash \bar{M}) \cap L_{\text {loc }}^{1}(G)$ is a function such

$$
\begin{gathered}
|(A-c) g(x)| \leq C_{0} \\
|\nabla g(x) \sigma(x)| \leq C_{0}
\end{gathered}
$$

in $G \backslash \bar{M}$ for some nonnegative constant $C_{0}$ and such that

$$
g(x) \rightarrow+\infty \text { if } x \in G \backslash \bar{M}, d(x, \bar{M}) \rightarrow 0 .
$$

Then for any point $p$ on $M$ there is an open neighborhood $U$ of $p$ in $G$ and a function $u$ of the class $L_{\text {loc }}^{1}(U)$ such that

$$
A u=0 \text { in } U
$$

and

$$
C_{1} g(x) \leq u(x) \leq C_{2} g(x) \quad \text { in } U \backslash M
$$

for some positive constants $C_{1}$ and $C_{2}$.

ProOF. Let $U$ and $V$ be open neighborhoods of a point $p \in M$ in $G$ such that $\bar{U} \subset V \subset G, \partial U$ is $C^{2}$-smooth and diameter $V$ is sufficiently small. By $\left(\Omega, \mathfrak{F}, \mathfrak{F}_{t}, x(t), P_{x}\right)$ we denote a diffusion process generated by the operator $\chi(A-c(x))$, where $\chi \in C_{0}^{\infty}(V)$ is a nonnegative function satisfying $\chi=1$ in $U$. We define a function $u(x)$ in $U \backslash M$ by

$$
\begin{aligned}
u(x) & =E_{x}\left[\chi g(x(\tau)) \exp \left\{\int_{0}^{\tau} \chi c(x(s)) d s\right\}\right] \\
& =E_{x}\left[g(x(\tau)) \exp \left\{\int_{0}^{\tau} c(x(s)) d s\right\}\right]
\end{aligned}
$$

where $\tau$ is the exit time of $x(t)$ from $\bar{U}$.

By Lemma 2.2 and (2.5), we have

$$
u(x)=g(x)+E_{x}\left[\int_{0}^{\tau} A g(x(t)) \exp \left\{\int_{0}^{t} c(x(s)) d s\right\} d t\right]
$$

and, by (2.4),

$$
\begin{aligned}
\left|E_{x}\left[\int_{0}^{\tau} A g(x(t)) \exp \left\{\int_{0}^{t} c(x(s)) d s\right\} d t\right]\right| \\
\leq C_{0} E_{x}\left[\tau e^{C \tau}\right]+C E_{x}\left[e^{C \tau} \int_{0}^{\tau} g(x(t)) d t\right]
\end{aligned}
$$


where $C=\sup _{x \in U} c(x) \vee 0$. Lemmas 2.2 and 2.3 give

$$
\begin{aligned}
E_{x}\left[e^{C \tau} \int_{0}^{\tau} g(x(t)) d t\right]= & E_{x}\left[e ^ { C \tau } \int _ { 0 } ^ { \tau } \left\{g(x)+\int_{0}^{t} \nabla g(x(s)) \sigma(x(s)) d w(s)\right.\right. \\
& \left.\left.+\int_{0}^{t}(A-c) g(x(s)) d s\right\} d t\right] \\
\leq & E_{x}\left[\tau e^{C \tau}\right] g(x)+\left(E_{x}\left[\left(\tau e^{C \tau}\right)^{2}\right]\right)^{1 / 2} \\
& \times\left(E_{x}\left[\sup _{0 \leq t \leq \tau}\left|\int_{0}^{t} \nabla g(x(s)) \sigma(x(s)) d w(s)\right|^{2}\right]\right)^{1 / 2} \\
& +C_{0} E_{x}\left[\frac{1}{2} \tau^{2} e^{C \tau}\right] \\
\leq & E_{x}\left[\tau e^{C \tau}\right] g(x)+C_{0} E_{x}\left[\tau e^{C \tau}\right]+2 C_{0} E_{x}\left[\left(\tau e^{C \tau}\right)^{2}\right] .
\end{aligned}
$$

Consequently, we obtain

$$
|u(x)-g(x)| \leq C E_{x}\left[\tau e^{C \tau}\right] g(x)+C_{0}(1+C) E_{x}\left[\tau e^{C \tau}\right]+2 C C_{0} E_{x}\left[\left(\tau e^{C \tau}\right)^{2}\right]
$$

This implies, by Lemma 1.7, $u \in L_{\text {loc }}^{1}(U)$ and (2.8).

Let us define functions $u_{n}(x), n \in \mathbf{N}$, in $U$ by

$$
u_{n}(x)=E\left[g_{n}(x(\tau)) \exp \left\{\int_{0}^{\tau} c(x(s)) d s\right\}\right],
$$

where $g_{n}(x)=g(x) \wedge n$. Lemmas 1.6 and 1.7 give

$$
\int u_{n} A^{*} \phi d x=0 \quad\left(\phi \in C_{0}^{\infty}(U)\right)
$$

By letting $n \rightarrow \infty$, we obtain (2.7).

LEMMA 2.5. Assume that $u \in L_{\text {loc }}^{1}\left(\mathbf{R}^{d}\right)$ is a function such that

$$
-C_{1} \log \left|x^{\prime \prime}\right| \leq u\left(x^{\prime}, x^{\prime \prime}\right) \leq-C_{2} \log \left|x^{\prime \prime}\right| \quad \text { in } \mathbf{R}_{x}^{d}
$$

for some positive constants $C_{1}$ and $C_{2}$, where $x^{\prime}=\left(x_{1}, \ldots, x_{r}\right), x^{\prime \prime}=\left(x_{r+1}, \ldots, x_{d}\right)$ and $0<r<d$. Then

$$
\left(0, \xi^{0}\right) \in W F(u) \quad \text { for some } \xi^{0} \in\left\{\xi \in \mathbf{R}^{d}:\left|\xi^{\prime}\right|=0\right\},
$$

where $\xi^{\prime}=\left(\xi_{1}, \ldots, \xi_{r}\right)$ and $\xi^{\prime \prime}=\left(\xi_{r+1}, \ldots, \xi_{d}\right)$.

Proof. We assume that $(0, \xi) \notin W F(u)$ when $\left|\xi^{\prime}\right|=0$. Then there is a nonnegative function $\chi \in C_{0}^{\infty}\left(\mathbf{R}^{d}\right)$ such that $\chi=1$ in a neighborhood of 0 in $\mathbf{R}^{d}$ and

for any fixed $\xi^{\prime}$. Hence

$$
\int_{\mathbf{R}_{x}^{d}} e^{-i x \cdot \xi} \chi(x) u(x) d x \in S\left(\mathbf{R}_{\xi^{\prime \prime}}^{d-r}\right)
$$

$$
\int_{\mathbf{R}_{x^{\prime}}^{r}} \chi\left(x^{\prime}, x^{\prime \prime}\right) u\left(x^{\prime}, x^{\prime \prime}\right) d x^{\prime} \in S\left(\mathbf{R}_{x^{\prime \prime}}^{d-r}\right) .
$$

On the other hand we have, by (2.9),

$$
\int_{\mathbf{R}_{x^{\prime}}^{r}} \chi\left(x^{\prime}, x^{\prime \prime}\right) u\left(x^{\prime}, x^{\prime \prime}\right) d x^{\prime} \rightarrow+\infty \quad \text { as }\left|x^{\prime \prime}\right| \rightarrow 0 .
$$

This is a contradiction. 
ProOF OF THEOREM 1. Without loss of generality, we may assume that $r=\operatorname{dim} M<d$ and $\bar{M} \cap V=\left\{x \in V: x_{r+1}=\cdots=x_{d}=0\right\}$ for some open neighborhood $V$ of $p$ in $G$. By (1) and (3) we have

$$
a_{i j}(x)=O\left(\left|x^{\prime \prime}\right|^{2}\right), \quad \sigma_{i j}(x)=O\left(\left|x^{\prime \prime}\right|\right), \quad b_{i}(x)=O\left(\left|x^{\prime \prime}\right|\right)
$$

for $r+1 \leq i, j \leq d$ when $x \in V \backslash \bar{M}$ and $d(x, \bar{M}) \rightarrow 0$, where $x^{\prime \prime}=\left(x_{r+1}, \ldots, x_{d}\right)$. Let us consider a function $g(x)=-\log \left|x^{\prime \prime}\right|$ defined in $V \backslash \bar{M}$. It is easy to show that $g(x)$ satisfies (2.4)-(2.6) for $G=V$. Hence, by Proposition 2.4, there is an open neighborhood $U$ of $p$ in $V$ and a function $u \in L_{\text {loc }}^{1}(U)$ such that (2.7) and (2.8) are valid. By Lemma $2.5,(2.8)$ implies that $\left(p, \xi^{0}\right) \in W F(u)$ for some $\xi^{0} \in\left(T_{p}^{*} M\right)^{\perp}$. The proof of Theorem 1 is complete.

\section{Proof of Theorem 2.}

Lemma $3.1[14,4]$. Assume $u \in D^{\prime}\left(\mathbf{R}^{d}\right)$ and $0 \in \operatorname{supp} u \subset\left\{x \in \mathbf{R}^{d}: x_{d} \geq 0\right\}$. Then

$$
\left(0, \xi^{0}\right) \in W F_{a}(u) \text { for some } \xi^{0} \in\left\{\xi \in \mathbf{R}^{d}:\left|\xi^{\prime}\right|=0\right\},
$$

where $\xi^{\prime}=\left(\xi_{1}, \ldots, \xi_{d-1}\right)$.

PROOF OF THEOREM 2. Let $U$ and $V$ be open neighborhoods of a point $p \in M$ in $G$ such that $\bar{U} \subset V \subset G, \partial U$ is $C^{2}$-smooth and diameter $V$ is sufficiently small. $\left(\Omega, \mathfrak{F}, \mathfrak{F}_{t}, x(t), P_{x}\right)$ denotes a diffusion process generated by the operator $\chi(A-c(x))$, where $\chi \in C_{0}^{\infty}(V)$ is a nonnegative function satisfying $\chi=1$ in $U$. Without loss of generality, we may assume $\bar{M} \cap V=\left\{x \in \mathbf{R}^{d}: x_{d}=0\right\}$ and, by (9), $\left\langle X_{0}(x), e_{d}\right\rangle<0$ on $\bar{M} \cap V$, where $e_{d}=(0, \ldots, 0,1)$. Let us take a function

$$
g(x)=\left(\begin{array}{ll}
e^{-1 /\left|x_{d}\right|} & \text { if } x_{d}>0 \\
0 & \text { if } x_{d} \leq 0
\end{array}\right.
$$

defined in $V$. By Lemmas 1.6 and 1.7,

$$
u(x)=E_{x}\left[g(x(\tau)) \exp \left\{\int_{0}^{\tau} c(x(s)) d s\right\}\right] \in L^{\infty}(U)
$$

is a solution of the Dirichlet problem

$$
A u=0 \quad \text { in } U, \quad \lim _{\substack{x \rightarrow a \\ x \in U}} u(x)=g(a) \quad(a \in \Sigma),
$$

where $\tau$ is the exit time of $x(t)$ from $\bar{U}$. Furthermore, by (7)-(9) and Lemma 1.8, we have

$$
u(x)\left(\begin{array}{ll}
>0 & \text { if } x_{d}>0 \\
=0 & \text { if } x_{d} \leq 0
\end{array}\right.
$$

Combining this fact with Lemma 3.1, we obtain the desired result.

4. Proof of Theorem 3. Throughout this section, we assume that $\psi \in C^{\infty}(G)$ is a real valued function such that $\psi=0$ at some point in $G, \nabla \psi \neq 0$ in $G$ and (11) is valid, and furthermore, we assume (12). Without loss of generality, we may assume that $\psi(x)=x_{d}$. We set $G_{+}\left\{x \in G: x_{d}>0\right\}, G_{-}=\left\{x \in G: x_{d}<0\right\}$ and $M=\left\{x \in G: x_{d}=0\right\}$. 
LEMMA 4.1. Given $u \in L^{\infty}(G)$ and $f \in L^{\infty}(G)$, if

$$
\begin{gathered}
\left|a_{d d}(x) u(x)\right|=o\left(\left|x_{d}\right|\right), \\
\left|\left(b_{d}(x)-\frac{\partial a_{d d}}{\partial x_{d}}(x)\right) u(x)\right|=o(1)
\end{gathered}
$$

uniformly in $x^{\prime}=\left(x_{1}, \ldots, x_{d-1}\right)$ as $x_{d} \rightarrow 0$ and if

$$
A u=f \text { in } G_{+} \cup G_{-} \text {, }
$$

then $A u=f$ in $G$.

PROOF. Let us take a function $\chi \in C_{0}^{\infty}(\mathbf{R})$ such that $0 \leq \chi \leq 1, \chi(t)=1$ when $|t| \leq 1 / 2$ and $\chi(t)=0$ when $|t| \geq 1$. For $\varepsilon>0$ we define a function $\chi_{\varepsilon} \in C^{\infty}\left(\mathbf{R}^{d}\right)$ by $\chi_{\varepsilon}(x)=\chi\left(x_{d} / \varepsilon\right)$. By (4.3), we easily have

$$
\int u(x) A^{*} \phi d x=\int f\left(1-\chi_{\varepsilon}\right) \phi d x+\int u A^{*} \chi_{\varepsilon} \phi d x
$$

for any $\varepsilon>0$ and any $\phi \in C_{0}^{\infty}(G)$. Direct computation gives

$$
\begin{aligned}
\int u A^{*} \chi_{\varepsilon} \phi d x= & \int_{\left|x_{d}\right| \leq \varepsilon} u a_{d d} \frac{\partial^{2} \chi_{\varepsilon}}{\partial x_{d}^{2}} \phi d x+2 \sum_{i=1}^{d} \int_{\left|x_{d}\right| \leq \varepsilon} u a_{i d} \frac{\partial \chi_{\varepsilon}}{\partial x_{d}} \frac{\partial \phi}{\partial x_{i}} d x \\
& -\int_{\left|x_{d}\right| \leq \varepsilon} u\left(b_{d}-\sum_{j=1}^{d} \frac{\partial a_{d j}}{\partial x_{j}}\right) \frac{\partial \chi_{\varepsilon}}{\partial x_{d}} \phi d x+\int_{\left|x_{d}\right| \leq \varepsilon} u \chi_{\varepsilon} A^{*} \phi d x .
\end{aligned}
$$

Since

$$
\frac{\partial \chi_{\varepsilon}}{\partial x_{d}}(x)=\frac{1}{\varepsilon} \chi^{\prime}\left(x_{d} / \varepsilon\right) \text { and } \frac{\partial^{2} \chi_{\varepsilon}}{\partial x_{d}^{2}}(x)=\frac{1}{\varepsilon^{2}} \chi^{\prime \prime}\left(x_{d} / \varepsilon\right),
$$

we obtain, by (4.1), (4.2) and (11),

$$
\lim _{\varepsilon \rightarrow 0} \int u A^{*} \chi_{\varepsilon} \phi d x=0 .
$$

Combining this fact with (4.4), we have $\int u A^{*} \phi d x=\int f \phi d x$ for any $\phi \in C_{0}^{\infty}(G)$.

Modifying the proof of Lemma 2.5, we easily have the following

LEMMA 4.2. Assume that $u \in L^{\infty}\left(\mathbf{R}^{d}\right)$ is a function such that

$$
u\left(x^{\prime}, x_{d}\right)\left(\begin{array}{ll}
\geq C_{1} & \text { if } x_{d}>0 \\
\leq C_{2} & \text { if } x_{d}<0
\end{array}\right.
$$

for some constants $C_{1}>C_{2}$, where $x^{\prime}=\left(x_{1}, \ldots, x_{d-1}\right)$. Then

$$
\left(0, \xi^{0}\right) \in W F(u) \text { for some } \xi^{0} \in\left\{\xi \in \mathbf{R}^{d}:\left|\xi^{\prime}\right|=0\right\},
$$

where $\xi^{\prime}=\left(\xi_{1}, \ldots, \xi_{d-1}\right)$.

Proposition 4.3. Assume that

$$
\left\langle X_{0}, e_{d}\right\rangle=0 \text { on } M
$$

where $e_{d}=(0, \ldots, 0,1) \in \mathbf{R}^{d}$, and assume that

$$
a_{d d}(x) / x_{d} \rightarrow 0 \text { as } x_{d} \rightarrow 0
$$


uniformly in $x^{\prime}=\left(x_{1}, \ldots, x_{d-1}\right)$. Then for any point $p \in M$ there is an open neighborhood $U$ of $p$ in $G$ and a function $u \in L^{\infty}(U)$ such that $A u=0$ in $U$ and

$$
u(x)\left(\begin{array}{l}
\geq 1 \text { in } U \cap G_{+}, \\
=0 \text { in } U \cap G_{-} .
\end{array}\right.
$$

ProOF. Let $U$ and $V$ be open balls with center $p \in M$ such that $\bar{U} \subset V \subset G$ and diameter $V$ is sufficiently small. By (11), (12), (4.6) and (4.7), we may assume that

$$
b_{1}(x) \neq 0 \text { in } V \text {. }
$$

(11) and (4.9) show that for any constant $C$ there are constants $\gamma_{1}$ and $\gamma_{2}$ such that

$$
a_{11}(x) \gamma_{1}^{2}+b_{1}(x) \gamma_{1}+C \geq 0 \text { in } V
$$

and

$$
a_{11}(x) \gamma_{2}^{2}+b_{1}(x) \gamma_{2}+C \leq 0 \text { in } V
$$

if diameter $V$ is sufficiently small. Here we note that $A u=0$ means

$$
A v+2 \gamma_{i} \sum_{j=1}^{d} a_{1 j}(x) \frac{\partial v}{\partial x_{j}}+\left(a_{11}(x) \gamma_{i}^{2}+b_{1}(x) \gamma_{i}\right) v=0
$$

when $u=e^{\gamma_{i} x_{1}} v$.

For $n \in \mathbf{N}$ we set $U_{n}=U \cap\left\{x \in G: x_{d}>1 / n\right\}$ and $V_{n}=V \cap\left\{x \in G: x_{d}>\right.$ $1 / 2 n\} . \quad\left(\Omega, \mathfrak{F}_{,}, \mathfrak{F}_{t}, x(t), P_{x}^{n}\right)$ denotes a diffusion process generated by the operator $\chi_{n}\left(A-c(x)\right.$ ) (cf. Lemma 1.4), where $\chi_{n} \in C_{0}^{\infty}\left(V_{n}\right)$ is a nonnegative function satisfying $\chi_{n}=1$ in $U_{n} . \tau_{n}$ is the exit time of $x(t)$ from $\bar{U}_{n}$. Applying Lemma 1.5 to functions $w_{i}(x)=e^{\gamma_{i} x_{1}}(i=1,2)$, we have, by (4.10)-(4.12),

$$
E_{x}^{n}\left[w_{1}\left(x\left(\tau_{n}\right)\right) e^{C \tau_{n}}\right] \geq w_{1}(x), \quad E_{x}^{n}\left[w_{2}\left(x\left(\tau_{n}\right)\right) e^{C \tau_{n}}\right] \leq w_{2}(x)
$$

in $U_{n}$ for any $n \in \mathbf{N}$. Hence, there are positive constants $C_{1}$ and $C_{2}$ independent of $n$ such that

$$
C_{1} \leq E_{x}^{n}\left[e^{C \tau_{n}}\right] \leq C_{2} \text { in } U_{n} .
$$

Lemma 1.6 and (4.13) show that, for each $n \in \mathbf{N}$,

$$
u_{n}(x)=\left(\begin{array}{ll}
E_{x}\left[\exp \left\{\int_{0}^{\tau_{n}} c(x(s)) d s\right\}\right] & \text { if } x \in U_{n}, \\
0 & \text { if } x \in U \backslash U_{n}
\end{array}\right.
$$

is a solution of the equation $A u=0$ in $U_{n} \cup\left(U \cap G_{-}\right)$such that

$$
C_{1}^{\prime} \leq u_{n}(x) \leq C_{2}^{\prime} \quad \text { in } U_{n}
$$

for some positive constants $C_{1}^{\prime}$ and $C_{2}^{\prime}$ independent of $n$. Since, by (4.14) and (4.15), $\left\{u_{n}: n \in \mathbf{N}\right\}$ is a bounded subset of the Hilbert space $L^{2}(U)$, there is a subsequence $\left\{u_{n_{i}}\right\}_{i=1}^{\infty}$ of $\left\{u_{n}\right\}_{n=1}^{\infty}$ and a function $u \in L^{2}(U)$ such that

$$
\lim _{i \rightarrow \infty} u_{n_{i}}=u \text { weakly in } L^{2}(U) \text {. }
$$


$A u=0$ in $U_{n} \cup\left(U \cap G_{-}\right)$means

$$
\int u_{n_{i}} A^{*} \phi d x=0 \text { for } \phi \in C_{0}^{\infty}\left(U_{n_{i}}\right) \cup C_{0}^{\infty}\left(U \cap G_{-}\right) .
$$

Combining (4.17) with (4.16), we have $A u=0$ in $U \cap\left(G_{+} \cup G_{-}\right)$. Hence, by (11), (4.6), (4.7) and Lemma 4.1, $A u=0$ in $U$. (4.15) implies that

$$
\int C_{1}^{\prime} \phi d x \leq \int u_{n_{i}} \phi d x \leq \int C_{2}^{\prime} \phi d x
$$

for any nonnegative function $\phi \in L^{2}\left(U_{n_{i}}\right)$. Letting $i \rightarrow \infty$ in (4.18), we obtain

$$
C_{1}^{\prime} \leq u(x) \leq C_{2}^{\prime} \quad \text { a.e. in } U \cap G_{+} .
$$

(4.14) and (4.16) easily give

$$
u(x)=0 \quad \text { a.e. in } U \cap G_{-} .
$$

Therefore, $u(x) / C_{1}^{\prime}$ is the desired solution.

Proposition 4.4. Assume that

$$
\left\langle X_{0}, e_{d}\right\rangle<0 \text { on } M \text {, }
$$

where $e_{d}=(0, \ldots, 0,1) \in \mathbf{R}^{d}$. Then for any function $g \in C^{2}(M)$ and any point $p \in M$ there is an open neighborhood $U$ of $p$ in $G$ and a function $u \in L^{\infty}(U)$ such that $A u=0$ in $U$ and

$$
\lim _{\substack{x \rightarrow a \\ x \in U \backslash M}} u(x)=g(a) \quad \text { for } a \in M \cap U
$$

ProOF. Let $U$ and $V$ be open balls with center $p \in M$ such that $\bar{U} \subset V \subset G$ and diameter $V$ is sufficiently small. Let us take an auxiliary function $w(x)=x_{d}^{\alpha}$, where $\alpha$ is a sufficiently small positive constant. By (11) and (4.21),

$$
(A-c(x)) w=\left\{\alpha\left(b_{d}-\frac{a_{d d}}{x_{d}}\right)+\alpha^{2} \frac{a_{d d}}{x_{d}}\right\} x_{d}^{\alpha-1} \rightarrow-\infty
$$

as $x_{d} \downarrow 0$. Hence, we may assume that

$$
A w \leq-1 \text { in } V
$$

and

$$
(A-c(x)+C)(w+1) \leq 0 \text { in } V,
$$

where $C=\sup _{x \in V} c(x) \vee 0$.

For $n \in \mathbf{N}$ we set $U_{n}=U \cap\left\{x \in G: x_{d}>1 / n\right\}$ and $V_{n}=V \cap\left\{x \in G: x_{d}>\right.$ $1 / 2 n\}$. $\left(\Omega, \mathfrak{F}, \mathfrak{F}_{t}, x(t), P_{x}^{n}\right)$ denotes a diffusion process generated by the operator $\chi_{n}(A-c(x))$, where $\chi_{n} \in C_{0}^{\infty}\left(V_{n}\right)$ is a nonnegative function satisfying $\chi_{n}=1$ in $U_{n}$. $\tau_{n}$ is the exit time of $x(t)$ from $\bar{U}_{n}$. Let $h(x)$ be a $C^{2}$ function defined in $V$ such that $h(x)=g(x)$ on $M \cap U$. Lemma 1.5 and (4.24) give

$$
E_{x}^{n}\left[\left(w\left(x\left(\tau_{n}\right)\right)+1\right) e^{C \tau_{n}}\right] \leq w(x)+1 \text { in } U_{n}
$$

this implies

$$
E_{x}^{n}\left[e^{C \tau_{n}}\right] \leq w(x)+1 \quad \text { in } U_{n}
$$


By (4.25) and Lemma 1.6, for each $n \in \mathbf{N}$,

$$
u_{n}(x)=\left(\begin{array}{l}
E_{x}^{n}\left[h\left(x\left(\tau_{n}\right)\right) \exp \left\{\int_{0}^{\tau_{n}} c(x(s)) d s\right\}\right] \quad \text { in } U_{n}, \\
0 \text { in }\left(U \cap G_{+}\right) \backslash U_{n}
\end{array}\right.
$$

is a solution such that

$$
A u_{n}=0 \text { in } U_{n}
$$

and

$$
\left|u_{n}\right| \leq \sup _{x \in U \cap G_{+}}|h(x)|(w+1) .
$$

Since, by (4.27), $\left\{u_{n}: n \in \mathbf{N}\right\}$ is a bounded subset of the Hilbert space $L^{2}\left(U \cap G_{+}\right)$, there is a subsequence $\left\{u_{n_{i}}\right\}_{i=1}^{\infty}$ of $\left\{u_{n}\right\}_{n=1}^{\infty}$ and a function $u_{+} \in L^{2}\left(U \cap G_{+}\right)$such that

$$
\lim _{i \rightarrow \infty} u_{n_{i}}=u_{+} \quad \text { weakly in } L^{2}\left(U \cap G_{+}\right) \text {. }
$$

(4.26)-(4.28) imply that $u_{+} \in L^{\infty}\left(U \cap G_{+}\right)$is a solution of the equation $A u=0$ in $U \cap G_{+}$. Lemma 1.5 and (4.23) give

$$
\begin{aligned}
u_{n}(x)-h(x)+C_{0} w(x) & \geq E_{x}^{n}\left[\int_{0}^{\tau_{n}} A h(x(t)) \exp \left\{\int_{0}^{t} c(x(s)) d s\right\} d t\right. \\
& \left.+\int_{0}^{\tau_{n}} C_{0} \exp \left\{\int_{0}^{t} c(x(s)) d s\right\} d t\right] \\
& \geq 0 \text { in } U_{n}
\end{aligned}
$$

and

$$
\begin{aligned}
u_{n}(x)-h(x)-C_{0} w(x) & \leq E_{x}^{n}\left[\int_{0}^{\tau_{n}} A h(x(t)) \exp \left\{\int_{0}^{t} c(x(s)) d s\right\} d t\right. \\
& \left.-\int_{0}^{\tau_{n}} C_{0} \exp \left\{\int_{0}^{t} c(x(s)) d s\right\} d t\right] \\
& \leq 0 \text { in } U_{n},
\end{aligned}
$$

where $C_{0}=\sup _{x \in U}|A h(x)|$. Hence, we have

$$
-\int C_{0} w \phi d x \leq \int\left(u_{n}-h\right) \phi d x \leq \int C_{0} w \phi d x
$$

for any nonnegative function $\phi \in L^{2}\left(U_{n}\right)$. Combining (4.29) with (4.28), we obtain

$$
\left|u_{+}-h\right| \leq C_{0} w \text { in } U \cap G_{+} \text {; }
$$

this implies

$$
\lim _{\substack{x \rightarrow a \\ x \in U \cap G_{+}}} u_{+}(x)=g(a) \quad \text { for } a \in M \cap U .
$$

For $n \in \mathbf{N}$ we see $U_{-n}=U \cap\left\{x \in G: x_{d}<-1 / n\right\}$ and $V_{-n}=V \cap\{x \in$ $\left.G: x_{d}<-1 / 2 n\right\}$. Let $\left(\Omega, \mathfrak{F}, \mathfrak{F}_{t}, x(t), P_{x}^{-n}\right)$ be a diffusion process generated by the operator $-\chi_{-n}(A-c(x))$, where $\chi_{-n} \in C_{0}^{\infty}\left(V_{-n}\right)$ is a nonnegative function 
satisfying $\chi_{-n}=1$ in $U_{-n}$. Then, by modifying the above argument, we can prove that there is a function $u_{-} \in L^{\infty}\left(U \cap G_{-}\right)$such that $A u_{-}=0$ in $U \cap G_{-}$and

$$
\lim _{\substack{x \rightarrow a \\ x \in U \cap G_{-}}} u_{-}(x)=g(a) \text { for } a \in M \cap U .
$$

Since

$$
u(x)=\left(\begin{array}{ll}
u_{+}(x) & \text { if } x \in U \cap G_{+}, \\
u_{-}(x) & \text { if } x \in U \cap G_{-}
\end{array}\right.
$$

is a solution of the equation $A(u-h)=-A h$ in $U \cap\left(G_{+} \cup G_{-}\right)$, we have, by (11), (4.30), (4.31) and Lemma 4.1, $A(u-h)=-A h$ in $U$, i.e., $A u=0$ in $U$. (4.30) and (4.31) easily give (4.22). The proof of Proposition 4.4 is complete.

PROOF OF THEOREM 3. Theorem 3(i) follows immediately from Proposition 4.3 and Lemma 4.2. In Proposition 4.4, if we take a function $g \in C^{2}(M)$ such that $p \in \operatorname{sing} \operatorname{supp} g$, then we have, by (4.22), $p \in \operatorname{sing} \operatorname{supp} u$. Thus Theorem 3(ii) follows from Proposition 4.4.

5. Proof of Theorem 4. Without loss of generality, we may assume that $\psi(x)=x_{d}$. We set $G_{+}=\left\{x \in G: x_{d}>0\right\}, G_{-}=\left\{x \in G: x_{d}<0\right\}$ and $M=\{x \in$ $\left.G: x_{d}=0\right\}$. Let $U$ and $V$ be open balls with center $p \in M$ such that $\bar{U} \subset V \subset G$ and diameter $V$ is sufficiently small. For $n \in \mathbf{N}$ we set $U_{n}=\left\{x \in U: x_{d}>1 / n\right\}$ and $V_{n}=\left\{x \in V: x_{d}>1 / 2 n\right\} . \quad\left(\Omega, \mathfrak{F}, \mathfrak{F}_{t}, x(t), P_{x}^{n}\right)$ denotes a diffusion process generated by the operator $\chi_{n}(A-c(x))$, where $\chi_{n} \in C_{0}^{\infty}(V)$ is a nonnegative function satisfying $\chi_{n}=1$ in $U_{n} . \tau_{n}$ is the exit time of $x(t)$ from $\bar{U}_{n}$.

Let us take a function

$$
h(x)=\left(\begin{array}{ll}
e^{-1 /\left|x_{d}\right|} & \text { if } x_{d}>0 \\
0 & \text { if } x_{d} \leq 0
\end{array}\right.
$$

Modifying the proof of Proposition 4.4, we can prove that

$$
u_{n}(x)=\left(\begin{array}{l}
E_{x}^{n}\left[h\left(x\left(\tau_{n}\right)\right) \exp \left\{\int_{0}^{\tau_{n}} c(x(s)) d s\right\}\right] \quad \text { in } U_{n}, \\
0 \quad \text { in } U \backslash U_{n}
\end{array}\right.
$$

are functions $\in L^{\infty}(U)$ such that $A u_{n}=0$ in $U_{n}$ and $\left\{u_{n}\right\}_{n=1}^{\infty}$ is uniformly bounded in $U$. Furthermore, we can show that there is a subsequence $\left\{u_{n_{i}}\right\}_{i=1}^{\infty}$ of $\left\{u_{n}\right\}_{n=1}^{\infty}$ and a function $u \in L^{\infty}(U)$ such that $A u=0$ in $U$ and

$$
\lim _{i \rightarrow \infty} u_{n_{i}}=u \text { weakly in } L^{2}(U) \text {. }
$$

(5.1) and (5.2) easily give supp $u \subset \overline{U \cap G_{+}}$.

Since $A u=0$ means

$$
\begin{aligned}
A v+ & 2 \gamma \sum_{i, j=1}^{d} a_{i j} \frac{\partial \psi}{\partial x_{i}} \partial \psi \partial x_{j} \\
& +\gamma\left\{\sum_{i, j=1}^{d} a_{i j}\left(\frac{\partial^{2} \psi}{\partial x_{i} \partial x_{j}}+\frac{\partial \psi}{\partial \psi_{i}} \frac{\partial \psi}{\partial x_{j}}\right)+\sum_{i=1}^{d} b_{i} \frac{\partial \psi}{\partial x_{i}}\right\} v=0
\end{aligned}
$$

when $u=e^{\gamma \psi} v$, we may assume, by (16) and (17), that

$$
c(x) \geq 0 \quad \text { in } U_{N}
$$


for any fixed $N$. We set

$$
F_{n}=\left\{\omega \in \Omega: x(t, \omega) \in \bar{U}_{N} \text { for all } t \leq \tau_{n}\right\}
$$

for $n \geq N+1$. It is easy to show that $\tau_{n}=\tau_{N}$ on $F_{N+1}$ and $F_{n}=F_{N+1}$ for any $n \geq N+1$. Since $\chi_{n}=1$ in $\bar{U}_{N}$ for $n \geq N+1$, we have

$$
\begin{aligned}
& E_{x}^{n}\left[\exp \left\{\int_{0}^{\tau_{n}} c(x(s)) d s\right\} \chi_{F_{n}}\right] \\
& =E_{x}^{N+1}\left[\exp \left\{\int_{0}^{\tau_{N+1}} c(x(s)) d s\right\} \chi_{F_{N+1}}\right]
\end{aligned}
$$

for $n \geq N+1$ and $x \in \bar{U}_{N}$. By (17) and Lemma 1.8 ,

$$
E_{x}^{N+1}\left[\exp \left\{\int_{0}^{\tau_{N+1}} c(x(s)) d s\right\} \chi_{F_{N+1}}\right]>0
$$

for $x \in \bar{U}_{N}$. Then we have

$$
\begin{aligned}
u_{n}(x) & \geq E_{x}^{n}\left[h\left(x\left(\tau_{n}\right)\right) \exp \left\{\int_{0}^{\tau_{n}} c(x(s)) d s\right\} \chi F_{n}\right] \\
& \geq\left(\inf _{y \in U_{N}} h(y)\right) E_{x}^{N+1}\left[\exp \left\{\int_{0}^{\tau_{N+1}} c(x(s)) d s\right\} \chi_{F_{N+1}}\right] \\
& >0
\end{aligned}
$$

for $x \in U_{N}$ and $n \geq N+1$; this implies that $u>0$ in $U_{N}$. Therefore, we have supp $u=\overline{U \cap G}_{+}$. By Lemma 3.1,u is the desired solution.

ACKNOWLEDGEMENT. The author would like to thank the referee for his helpful criticisms and suggestions.

\section{REFERENCES}

1. K. Amano, A necessary condition for hypoellipticity of degenerate elliptic-parabolic operators, Tokyo J. Math. 2 (1979), 111-120.

2. Equations 6 (1981), 903-916.

3. R. Beals and C. Fefferman, On hypoellipticity of second onder operators, Comm. Partial Differential Equations 1 (1976), 73-85.

4. J.-M. Bony, Equivalence des diverses notions de spectre singutier anabytique, Séminaire GoulaouicSchwartz, 1976/77.

5. M. Derridj, Sur une class d'opérateurs différentiels hypoelliptiques à coefficients analytiques, Séminaire Goulaouic-Schwartz, 1970/71.

6. A. Friedman, Stochastic differential equations and applications. I, II, Academic Press, New York, $1975,1976$.

7. B. Helffer and C. Zuily, Non-hypoellipticité d'une classe d'opénateurs différentiels, C.R. Acad. Sci. Paris Sér. A-B 277 (1973), 1061-1063.

8. L. Hörmander, Pseudo-differential operators and hypoelliptic equations, Proc. Sympos. Pure Math., vol. 10, Amer. Math. Soc., Providence, R.I., 1967, pp. 138-183.

9. __ Hypoeltiptic second onder diffenential equations, Acta Math. 119 (1967), 147-171.

10. Y. Kannai, Hypoeltiptic ondinary differential operators, Israel J. Math. 13 (1972), 106-134.

11. S. Kusuoka and D. W. Stroock, Applications of the Malliavin calculus. I, II, III (to appear).

12. O. A. Oleĭnik and E. V. Radkevix, Second onder equations with nonnegative characteristic form, Plenum, New York, 1973.

13. R. S. Phillips and L. Sarason, Eltiptic-parabolic equations of second order, J. Math. Mech. 17 (1967), 891-917. 
14. M. Sato, M. Kashiwara and T. Kawai, Microfunctions and pseudo-differential equations, Lecture Notes in Math., vol. 287, Springer-Verlag, Berlin and New York, 1972, pp. 265-529.

15. D. Stroock and S. R. S. Varadhan, On degenerate elliptic-parabolic operators of second onder and their associated diffusions, Comm. Pure Appl. Math. 25 (1972), 651-713.

16. H. J. Sussmann, Orbits of families of vector fields and integrability of distributions, Trans. Amer. Math. Soc. 180 (1973), 171-188.

17. C. Zuily, Sur l'hypoelipticité des opérateurs différentiels d'ondre 2 à coefficients réels, C.R. Acad. Sci. Paris Sér. A-B 277 (1973), 529-530.

Department of Mathematics, Josai University, SAKado, Saitama, Japan 